\title{
An Unusual Presentation of Acute Subdural Hematoma Secondary to Disseminated Intravascular Coagulation Following Conservative Management of Placenta Increta
}

\author{
Muhammad Fairuz Abdul Rahman ${ }^{\mathrm{a}, \mathrm{c}}$, Ghee Kheng Chew ${ }^{\mathrm{b}}$, \\ Eng Loy Tan ${ }^{\mathrm{a}}$, Wei Ching Tan ${ }^{\mathrm{a}}$
}

\begin{abstract}
With increasing cesarean section rates around the world, there will be a corresponding rise in morbidly-adherent placenta cases, such as placenta increta. There have been no randomized controlled trials comparing different surgical treatment of placenta increta. Conservative management of placenta increta, though largely successful, can potentially lead to significant maternal morbidity, such as sepsis, hemorrhage, and mortality. We report a rare event of disseminated intravascular coagulation (DIVC) with spontaneous maternal subdural hematoma (SDH) arising from the conservative management of placenta increta. To our knowledge, there has been no such case reported in the literature. The patient was managed by a multidisciplinary team of gynecologists, anesthesiologists, neurosurgeons and hematologists when she presented with right-sided progressive headache and extensive bruising 9 weeks postpartum. Blood products were given to correct her hemodynamic status and she subsequently underwent an emergency hysterectomy, with resolution of the DIVC and her symptoms.
\end{abstract}

Keywords: Acute subdural hematoma; Disseminated intravascular coagulation; Placenta increta

\section{Introduction}

With rising cesarean section rates around the world, combined with increasing maternal age, we are witnessing an increase in the incidence of morbidly-adherent placenta and its complications [1]. Several recognized risk factors for morbidlyadherent placenta include termination of pregnancy, intrau-

Manuscript submitted December 12, 2017, accepted December 14, 2017

aDepartment of Obstetrics \& Gynecology, Singapore General Hospital, Singapore

bepartment of General/Gynecological Oncology Surgery, Penang Adventist Hospital, Malaysia

${ }^{c}$ Corresponding Author: Muhammad Fairuz Abdul Rahman, Department of Obstetrics \& Gynecology, Singapore General Hospital, Outram Road, 169608, Singapore. Email: muhammadfairuzb.abdulrahman@mohh.com.sg

doi: https://doi.org/10.14740/jmc2989w terine surgery, smoking, multi-fetal gestation, and increasing parity, apart from previous cesarean section and advance maternal age. A morbidly-adherent placenta is a condition when part of the placenta, or the entire placenta, invades through the decidua basalis into and through the myometrium [2]. This includes placenta accreta, increta and percreta. Researchers quote an incidence rate of 1 in 533 pregnancies for placenta accreta for the period of 1982 - 2002, compared to a rate of 1 in 4,027 pregnancies in the $1970 \mathrm{~s}$, and 1 in 2,510 pregnancies in the 1980s [3-5].

Placenta increta is an intermediate in the spectrum of abnormal placental villous implantation, where the placental villi extend beyond the confines of the endometrium and invade the myometrium. There are no randomized controlled trials to determine the most effective surgical management of placenta increta. For patients desiring future fertility, the conservative approach where the placenta is kept in situ at the time of cesarean section is fraught with potential complications, such as infection and hemorrhage [6]. Disseminated intravascular coagulation (DIVC) occurs in about 7\% of such cases, and more often than not, patients end up requiring a hysterectomy for definitive treatment [7].

To date, there has been no reported case in the literature of a conservative management of placenta increta who presents with the primary diagnosis of subdural hematoma (SDH). A PubMed search using the Medical Subject Headings (MeSH) "placenta increta", "subdural hematoma" and "disseminated intravascular coagulation" yielded no matches. We present the first case of acute SDH secondary to DIVC as a result of the conservative management of placenta increta.

\section{Case Report}

Madam M is a multigravida with one previous lower segment cesarean section for breech presentation. She had no other significant medical history. She received routine antenatal care and was diagnosed with placenta previa major from the 20week detailed ultrasound screening scan. Subsequent ultrasound scans showed a normally growing baby and low suspicion of placenta accreta. She underwent an elective cesarean section at term.

Intra-operatively, the lower uterine segment appeared to be bulging with placenta invading the myometrium and en- 


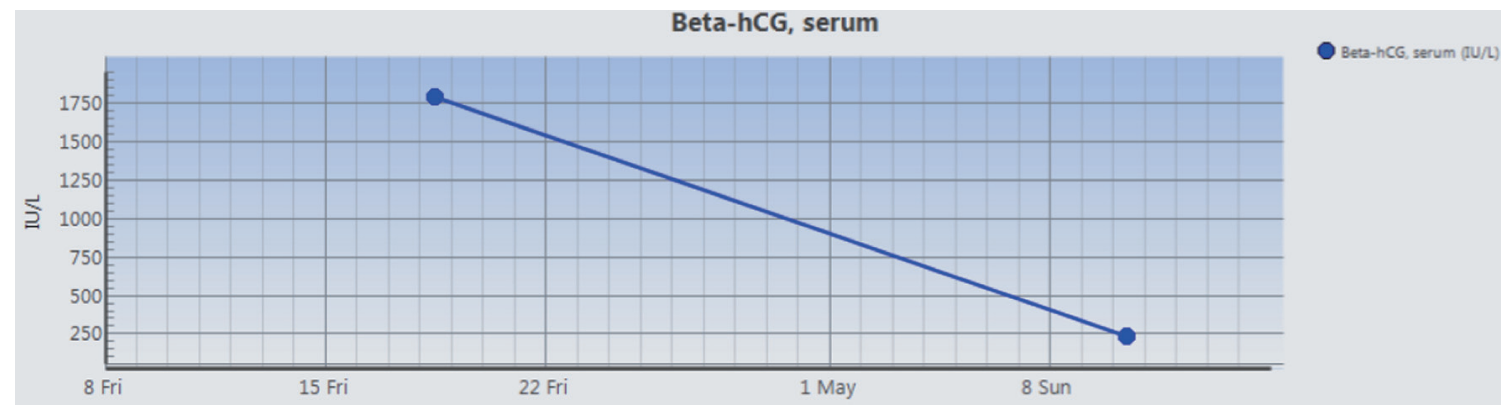

Figure 1. Postpartum trend of $\beta$-hCG levels.

gorged blood vessels, raising the suspicion of placenta increta. A classical fundal incision was made to deliver the baby, and the uterus was repaired in three layers. A decision to leave the placenta in situ was made in view of minimal bleeding and no sign of placental separation.

Madam M was discharged well on post-operative day 4. She received intramuscular methotrexate 2 weeks postpartum which led to a gradual decline in her $\beta$-human chorionic gonadotropin ( $\beta$-hCG) levels (Fig. 1).

However, Madam M presented to the emergency department at 9 weeks postpartum with severe progressive right-sided headache and extensive right lower limb bruises. Examination revealed extensive ecchymoses on her limbs, and a large

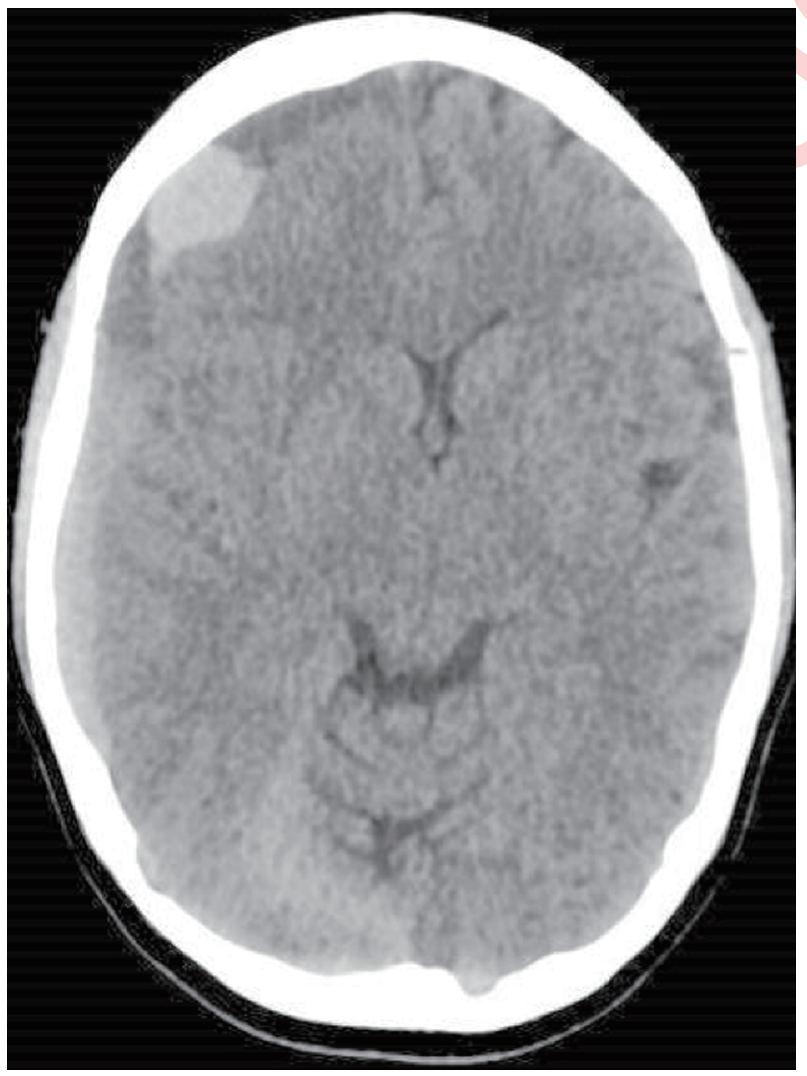

Figure 2. A CT scan of the brain at presentation. Acute subdural hematoma (SDH) overlying the right cerebral convexity, associated with mass effect and left-ward midline shift. firm abdominopelvic mass up to the level of the umbilicus.

A computed tomography (CT) brain scan (Fig. 2) showed an acute SDH with midline shift. A CT scan of the abdomen and pelvis (Fig. 3) showed a markedly hypervascular mass within the uterus with dilated and tortuous vessels, with no evidence of peritoneal hematoma.

She was confirmed to be in DIVC when laboratory investigations showed thrombocytopenia, prolonged prothrombin time (PT), and prolonged partial thromboplastin time (PTT), as well as elevated D-dimer and low fibrinogen level (Table 1).

A multidisciplinary team meeting of consultant obstetri-

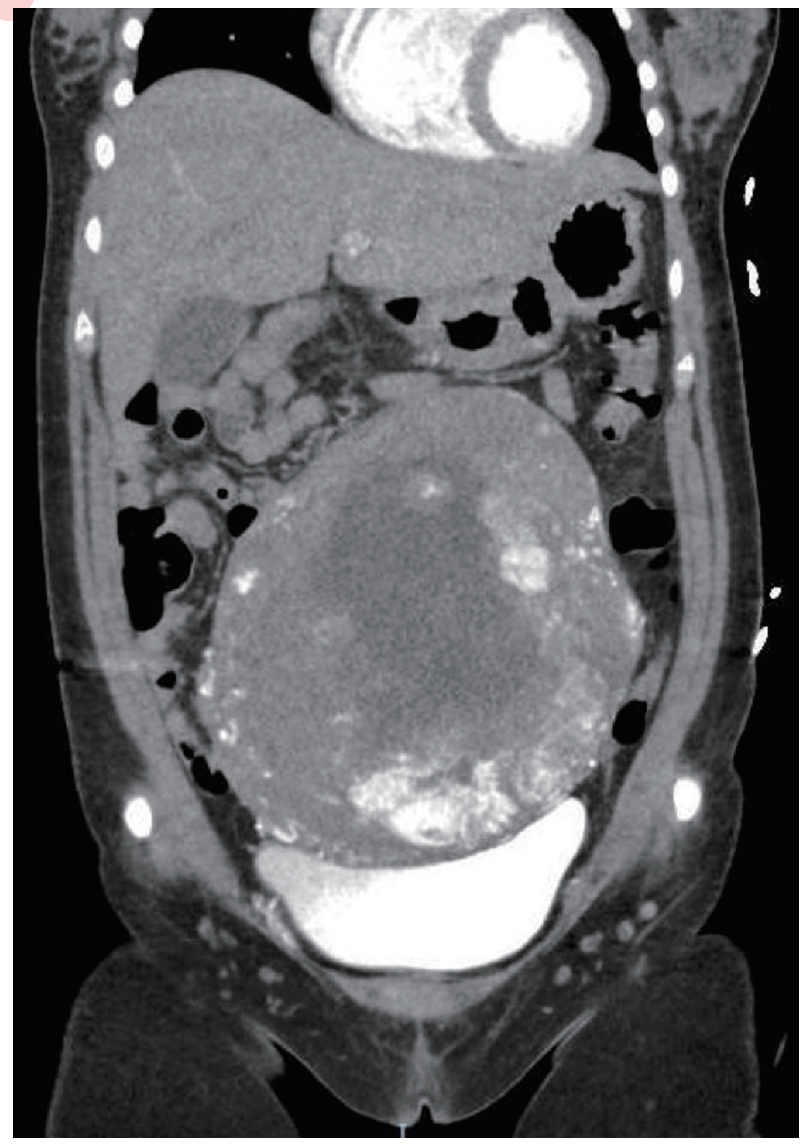

Figure 3. A CT scan of the abdomen and pelvis. Enlarged uterus extending above the umbilicus. Markedly hypervascular mass within the uterus with dilated and tortuous vessels coursing the mass. 
Table 1. Pre-Operative Laboratory Values

\begin{tabular}{ll}
\hline Hemoglobin & $11.5 \mathrm{~g} / \mathrm{dL}$ \\
WBC count & $6.19 \times 10^{9} / \mathrm{L}$ \\
Platelet & $146 \times 10^{9} / \mathrm{L}$ \\
PTT & $39.2 \mathrm{~s}$ \\
PT & $16.8 \mathrm{~s}$ \\
Fibrinogen & $0.49 \mathrm{~g} / \mathrm{L}$ \\
D-dimer & $>32.00 \mathrm{mg} / \mathrm{L} \mathrm{FEU}$ \\
\hline
\end{tabular}

WBC: white blood cell; PTT: partial thromboplastin time; PT: prothrombin time.

cians and gynecologists, neurosurgeon, hematologist and anesthetist convened to plan the management. Multiple blood products including cryoprecipitate were administered. When coagulation status was stable, Madam $\mathrm{M}$ underwent a burr hole drainage of the SDH and emergency hysterectomy. Her DIVC resolved within $24 \mathrm{~h}$ postoperatively (Table 2). Histology confirmed the diagnosis of placenta increta. A repeat CT brain scan showed improvement in mass effects (Fig. 4).

Madam M was discharged well subsequently and continued her outpatient rehabilitation. She had no significant residual neurological deficits.

\section{Discussion}

In conclusion, the conservative management of placenta increta may be complicated by DIVC with potentially debilitating consequences for the patient. These patients should be closely followed up postpartum with serial investigations, ideally till there is evidence of complete resolution of placenta increta from imaging studies and until serum $\beta$-hCG becomes undetectable. However, there is a lack of clear guidance on the optimal follow-up protocol for such cases. In the absence of available guidance of the conservative treatment of placenta increta, management plan needs to be individualized with ready access to tertiary hospital care. This case of acute maternal SDH secondary to DIVC due to the conservative management of placenta increta is probably the first reported case in literature. DIVC predisposes one to bleeding, which may be obvious (such as per vaginal bleeding) or occult, such as in this case, where bleeding occurred in the subdural compartment and the skin. As complications secondary to conservative man-

Table 2. Resolution of DIVC Following Surgery

\begin{tabular}{ll}
\hline Hemoglobin & $9.3 \mathrm{~g} / \mathrm{dL}$ \\
WBC count & $8.05 \times 10^{9} / \mathrm{L}$ \\
Platelet & $184 \times 10^{9} / \mathrm{L}$ \\
PTT & $26.2 \mathrm{~s}$ \\
PT & $10.1 \mathrm{~s}$ \\
Fibrinogen & $3.19 \mathrm{~g} / \mathrm{L}$ \\
\hline
\end{tabular}

WBC: white blood cell; PTT: partial thromboplastin time; PT: prothrombin time.

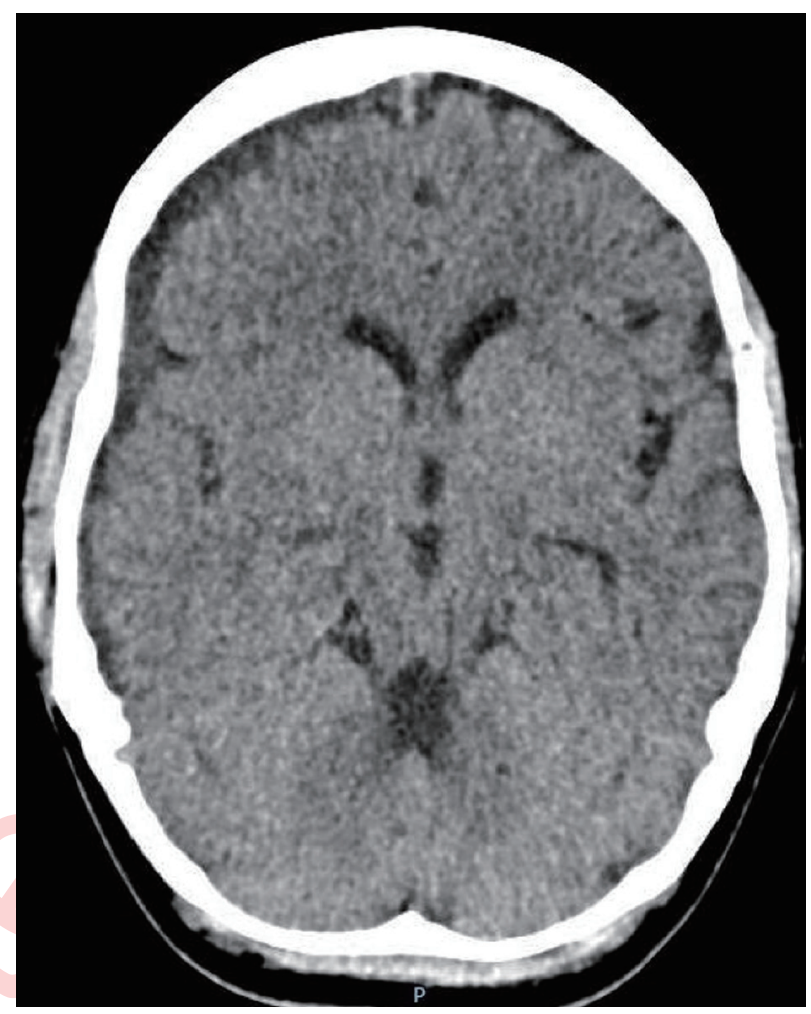

Figure 4. A CT scan of the brain 3 weeks post-hysterectomy. Interval expected evolution of previously noted intracranial hemorrhages with improvement in mass effects and left sided midline shift.

agement of morbidly-adherent placenta will likely mirror rising cesarean section rates, early recourse to cesarean hysterectomy should be considered as the definitive surgical treatment, especially for women who have no desire for future fertility.

\section{References}

1. Oyelese Y, Smulian JC. Placenta previa, placenta accreta, and vasa previa. Obstet Gynecol. 2006;107(4):927-941.

2. RCOG Green-top Guideline No. 27: Placenta praevia placenta praevia accreta and vasa praevia: diagnosis and management (January 2011).

3. Wu S, Kocherginsky M, Hibbard JU. Abnormal placentation: twenty-year analysis. Am J Obstet Gynecol. 2005;192(5):1458-1461.

4. Read JA, Cotton DB, Miller FC. Placenta accreta: changing clinical aspects and outcome. Obstet Gynecol. 1980;56(1):31-34.

5. Miller DA, Chollet JA, Goodwin TM. Clinical risk factors for placenta previa-placenta accreta. Am J Obstet Gynecol. 1997;177(1):210-214.

6. Eller AG, Porter TF, Soisson P, Silver RM. Optimal management strategies for placenta accreta. BJOG. 2009;116(5):648-654.

7. Timmermans S, van Hof AC, Duvekot JJ. Conservative management of abnormally invasive placentation. Obstet Gynecol Surv. 2007;62(8):529-539. 